\title{
Zur Mystik des Brahmanismus-Hinduismus
}

\author{
Ein Diskussionsbeitrag \\ Von PENTTI AALTO
}

Es gibt innerhalb der indischen Religion zwei Sekten, die einen ausgeprägt mystischen Charakter besitzen. Die Tantristen, deren Literatur und Gedankenwelt im Westen vor allem durch John Woodroffe alias Arthur Avalon bekannt gemacht worden sind, wollen die Erlösung durch sakramentalen Gebrauch des Verbotenen (madya ,Rauschgetränke', mämsa ,Fleisch', matsya ,Fisch', mudrã ,geröstetes Korn', maithuna ,Begattung') erreichen. Die nordindische mystische Bewegung, deren vornehmste Vertreter Rāmānanda, Kabīr und Dādu sind, verdankt ihr Dasein wenigstens zum Teil dem Einfluss des Sufismus. Sie ist von K. M. Sen in seinem Medieval Mysticism in India (London 1936) dargestellt worden ${ }^{1}$.

Ich lasse aber diese zwei hier beiseite, weil sie allgemein bekannt sein dürften, und ihr mystischer Charakter ohne weiteres klar ist, und begrenze mich auf einige Bemerkungen über m. E. mystische Züge in den Anschauungen einiger anderer indischen religiösen Richtungen.

Alle indischen orthodoxen Systeme erkennen die Autorität des Veda an. Im Veda ist aber vieles nicht mit Sicherheit verständlich, und scheint schon von den einheimischen Kommentatoren oft genug unzutreffend aufgefasst und erläutert worden zu sein. Die Vedareligion wird im allgemeinen als eine den praktischen Bedürfnissen des menschlichen Lebens dienende, vom Brahmanenstande verwaltete Ritualreligion beschrieben. Gewisse Einzelheiten, z. B. das rituelle Soma-Getränk, haben Entsprechungen in der vorzarathustrischen Religion Irans. Soma wurde nach dem Veda aus einer gewissen Pflanze durch Pressung bereitet, es ist aber unbekannt, aus welcher, und dasselbe gilt auch von seiner iranischen Entsprechung haoma $a^{2}$. Gleicher-

${ }^{1}$ Fernere Literatur bei Gonda, Die Religionen Indiens II, Stuttgart I963, S. $97 \mathrm{ff}$. u. I7o ff.

${ }^{2}$ Vgl. mein „Madyam apeyam“, F̃̃̄anamuktāvalī (Sarasvati Vihara Series No.

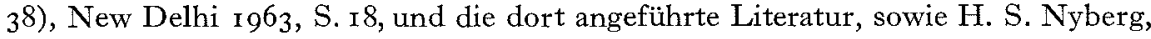
Die Religionen des alten Iran, Osnabrück 1966, S. 83. 
weise ist es unklar, wie soma den in den Hymnen so hoch gepriesenen RauschZustand verursachte. Aus den Zubereitungsvorschriften zu schliessen kann es z. B. nicht viel Alkohol enthalten haben. Auf Grund der Angaben in vedischer Ritual-Literatur, vor allem im Drāhyäyanaśrautasütra, dessen Übersetzung eben von Dr. Asko Parpola in Helsinki in Druck gegeben worden ist, möchte ich die Hypothese in Erwägung ziehen, dass das im Ritual gepresste und getrunkene soma nur symbolisch war, und erst durch die zu rezitierenden Mantras mit dem „himmlischen“ soma, dem Göttertrunk, mystisch identisch wurde: nur das soma der Götter besass die in den Texten gepriesenen Eigenschaften. Es handelte sich also um ein Mysterium, durch das der Mensch, vor allem der Offiziant, mit dem Göttlichen in Berührung kommen konnte. Die irrationale Natur des Soma kommt m. E. auch deutlich zum Ausdruck bei seinem angeblichen Gebrauch als Arzneimittel in der indischen Medizin'.

Das ganze vedische Opferwesen dürfte $\mathrm{m}$. E. weniger rational aufzufassen sein als es öfters dargestellt wird. Die im Opfer inhärente Kraft wird z. B. durch zeremonielle Handlungen auf den Priester und den Opferer übertragen, die somit eine mystische Gemeinschaft bilden. Es ist in ähnlichen Fällen oft schwer, einen klaren Unterschied zwischen Mystik und Magie zu machen. Die im Opfer innewohnende Kraft ist bráhman, der vielleicht am schwersten auffassbare und tiefsinnigste Begriff im ganzen indischen Denken.

Die ganze nachvedische religionsgeschichtliche Entwicklung in Indien könnte man als eine Anpassung des indogermanischen Erbes der Arier an das urindische, d. h. wahrscheinlich drawidische, Substrat betrachten. Wie Gonda (II S. 16) bemerkt, müssen die einwandernden Arier schon mit Mystik vertraut gewesen sein, und es dürfte kaum möglich sein, zu unterscheiden, was in der späteren Gedankenwelt von dem arischen Erbe bzw. von dem urindischen Substrat herstammt.

Die Upanișaden sind oft als eine Art Protest gegen den Ritualismus der Veda-Religion gedeutet worden. In ihnen spiegelt sich jedenfalls eine offenbar neue Tendenz wider: der unmittelbare Kontakt mit dem Göttlichen wird im Inneren des Menschen gesucht. Es wird oft darauf hingewiesen, dass es

\footnotetext{
${ }^{1}$ Vgl. die Úbersetzung des betreffenden Kapitels in Suśruta von F. G. Müller, Asiatica (Festschrift Friedrich Weller), Leipzig I954, S. 428 ff. Vgl. ferner H. Lommel, ,König Soma“, Numen II, Leiden I 955, S. I96-205.
} 
sich in den Upanișaden um „Wissen“ bzw. „Weisheit" oder sogar um „Wissenschaft" und nicht um „Religion" handele, und tatsächlich beschreiben die Texte selbst regelmässig ihre Botschaft mit Wörtern wie jñanna, vidy $\bar{a}$ usw. Es ist aber wichtig zu beachten, dass diese Sanskrit-Ausdrücke an sich kein ,objektives Wissen" sondern eben ein auf einem inneren Erlebnis beruhendes Wissen bezeichnen; z. B. jñãna wird bei Apte erklärt ,,sacred knowledge, especially knowledge derived from meditation on the higher truths of religion and philosophy, which teaches man how to understand his own nature and how he may be reunited to the Supreme Spirit“. Radhakrishnan bemerkt in der Einleitung seiner Upanișad-Ausgabe ${ }^{1}$, dass die Welt an sich nicht real ist, und dass die Realität nur im Inneren des Menschen zu finden ist: eben dieses innere Wissen wird in den Texten vidy $\bar{a}$ bezeichnet. Das Gleiche kann man indirekt durch die Erklärungen des Gegenbegriffes avidyä herausfinden.

In seinem Aufsatz „Upanișad“2 wollte Oldenberg die Bedeutung dieses wichtigen und viel diskutierten Wortes mit „Verehrung“ wiedergeben, erklärte aber dann den Sinn der Upanişad als ,,die Anweisung, in welcher Form, unter welchem Namen oder konkretem Bilde der Fromme ... mit seinen Gedanken den höchsten, verborgensten Objekten der Anbetung nahen soll“". In einer Fussnote zu seiner Śatapathabrähmana-Übersetzung XII 2, 2, 23 ( $S B E$ XLIV S. 155) bemerkt Eggeling, dass „Meditation“ eine angemessenere Übersetzung sein dürfte als die von Oldenberg vorgeschlagene „Verehrung“'3. Die gewöhnliche Deutung „Geheimlehre“ wurde dann von St. Schayer ${ }^{4}$ verworfen. Es scheint mir, dass Oldenberg seine umschreibende Erklärung des Sinnes besser gelungen ist als seine Übersetzung „Verehrung“. Dementsprechend schlug auch Schayer (S. 6r f.) die Deutung ,ma-

${ }_{1}$ The Principial Upanișads, London 1953.

2 Ursprünglich ein Vortrag vor dem Stockholmer Orientalistenkongress ז889, erstmalig veröffentlicht in $Z D M G 50,1896$, jetzt wieder Kleine Schriften, Wiesbaden I 967 , I S. 35 ff.; vgl. ferner ibid. II S. I320, , On the oldest form of the Upanishads".

${ }^{3} \mathrm{Vgl}$. ferner die Ansicht von Max Müller in seinem ,,Meaning of the Word Upanishad" in $S B E$ I S. Lxxix ff.

${ }^{4}$,Ủber die Bedeutung des Wortes upanișad“, Rocznik Orjentalistyczny III, Lwów 1925, S. 57: „Die Ubersetzung des Wortes upanișad als „Geheimlehre“ ist daher, sofern es sich um die älteren Texte handelt, nichts anderes als eine willkürliche Etymologie“. 
gische Äquivalenz", „symbolische Identität" vor, und tatsächlich lassen sich zahlreiche eindeutige Fälle und genuine Glossierungen des Wortes upanişad finden, die eben für die Bedeutung ,Gleichung, Äquivalenz, Identifikation"1 zu sprechen scheinen, z. B. SB XII 2, 2, I3 ahar iti sarvam samvatsaram saișā samvvatsarasya-upanișat „, der Tag ist das Jahr, er ist die upanișad des Jahres", Pānini I 4, 79 jīvikā-upanișadau-aupamye ,jūikā (und) upanișad (sind gati, wenn verbunden mit dem Verb kr-) in der Bedeutung „Analogie“", in der tibetischen Übersetzung des Vajracchedikā 16 b 24 wird upanișad durch rgyu, Vergleich, Gleichung, Identifikation' wiedergegeben, usw. Das, was die Upaniṣaden namentlich lehren wollen, ist ja die Identität des ätman mit dem bráhman, z. B. Br̆hadäranyaka-Upanișad II 5, I4 ayam sa yo 'yam ătmā, idam amrtam, idam brahma, idam sarvam, er ist eben dieses ātman, dies ist amrtam, dies ist brahman, dies ist All', Chändogya-Upanișad VIII I4, I te yad antarā tad brahma, tad amrtam, sa ātmā, das, innerhalb dessen sie sind, ist brahman, dies ist amrtam, er ist ätman', usw. Die Brahmasütras sagen auch ausdrücklich 3, 2, 23 tadaryaktam-āha hi, dies (brahman ist) unmanifestiert, sagt (die Offenbarung) ${ }^{\complement}, 24$ api ca samrāadhane pratyakṣānumānābhyãm, und ferner (wird brahman erlebt) in vollständiger Meditation (wie wir wissen) durch die Offenbarung und Tradition', und die S'vetāśvataraUpanișad sagt IV $19-20$ (in Übersetzung von Radhakrishnan S. 736) „Not above, not across, not in the middle, nor has any one grasped Him. There is no likeness of Him whose name is great glory. His form is not to be seen; no one sees Him with the eye. Those who through heart and mind know him abiding in the heart become immortal".

Auch in dem Yoga-System kann man einen Versuch sehen, vor allem mit Hilfe körperlicher Konzentration, ein unmittelbares Erlebnis von der Identität des Ichs mit dem Göttlichen zu erreichen. Auch das Wort yoga könnte in einer dem Namen upanișad entsprechenden Weise gedeutet werden; so sagt Bhagavadgïtã II 48 samatvam yoga ucyate, Gleichheit Identität wird yoga genannt', und Sankara glossiert Chāndogya-Upanișad I I, Io upanișadā durch yogena.

${ }^{1}$ Der Erklärung Schayers schliessen sich an u. a. Renou (Louis Renou et Jean Filliozat, L'Inde classique I, Paris I947, S. 299 § 590) und Nils Simonsson (Indisk filosofi: Sānkhya, Stockholm 1955, S. 7). 
Monier-Williams ${ }^{1}$ wollte die indische Religion in ihrem pantheistischen Aspekt Brahmanismus, in ihrem polytheistischen Aspekt wieder Hinduismus nennen. Gewöhnlich wird aber die historisch ältere Entwicklungsstufe Brahmanismus, die jüngere wieder Hinduismus genannt. Chronologisch müsste wohl Bhagavadgitta zu dem vorigen gerechnet werden, es ist aber fortwährend ein grundlegendes Werk für das religiöse Denken in Indien. Es enthält die bekannte grossartige Beschreibung des mystischen Erlebnisses: XI 9-13 (in der Übersetzung von Radhakrishnan):

„Having thus spoken ... Hari, the great lord of yoga, then revealed to Pärtha His Supreme and Divine Form, of many mouths and eyes, of many visions of marvel, of many divine ornaments, of many divine uplifted weapons, wearing divine garlands and raiments, with divine perfumes and ointments, made up of all wonders, resplendent, boundless, with face turned everywhere. If the light of a thousand suns were to blaze forth all at once in the sky, that might resemble the splendour of that exalted Being. There the Pandava beheld the whole universe, with its manifold divisions gathered together in one, in the body of the God of gods."

Einen viel neueren und zugleich recht interessanten Beleg für das Erlebnis des Göttlichen finden wir in der Ramakrishna-Biographie von Romain Rolland2:

„,Un jour, j'étais en proie à une intolérable angoisse. Il me semblait qu'on me tordait le cœur comme un linge mouillé ... La souffrance me déchirait. A l'idée que je n'aurais pas dans ma vie la bénédiction de cette vision divine, une frénésie terrible me saisit. Je pensai: Si cela doit être ainsi, assez de cette vie! ... La grande épeé pendait dans le sanctuaire de Kâlî. Mon regard tomba sur elle; et j'eus le cerveau traversé d'un éclair. - Elle! Elle m'aidera à mettre fin ... Je me précipitai. Je l'empoigne, comme un fou ... Et voici! La pièce, avec toutes ses portes et ses fenêtres, le temple tout s'évanouit. Il me sembla que plus rien n'existait. Et, à la place, je perçus un océan d'esprit, sans limites, éblouissant. De quelque part que je tournasse les yeux, aussi loin que je regardais, je voyais arriver d'énormes vagues de cet océan luisant. Elles se précipitaient furieusement sur moi, avec un bruit formidable, comme pour m'avaler. En un instant elles furent sur moi, elles s'écroulèrent, elles m'engouffrèrent. Roulé par elles, je suffoquai. Je perdis conscience, et je tombai ...

1 Hinduism, London I9r I, S. г 3.

- Essai sur la mystique et l'action de l'Inde vivante: I. La vie de Ramakrishna, Paris I 929 , S. 45 ff. 
Comment passèrent ce jour et le suivants, je ne sais point. Au dedans de moi roulait un océan de joie ineffable. Et jusqu'au fond, j'étais conscient de la présence de la Divine Mère ..."

Sehr interessant ist in der Fortsetzung die Beschreibung Rollands, wie der erlebte formlose Ozean die Gestalt der Göttin annimmt (S. $4^{8}$ f.):

„... il comprit ... C'était la Mère qui le possédait ... Elle le remplit. Et de la brume, la personne matérielle de la Déesse, morceau par morceau, émergea. Ce fut d'abord sa main entrevue — son souffle — sa voix — puis elle, tout entière ... II finissait par s'identifier à elle ... Au milieu des actes rituels, il était pris de pertes de conscience, d'engloutissements, de pétrifications, où il percevait le verrouillement des gonds de ses jointures, qui le murait à l'intérieur d'une statue“, usw.

Beide Beschreibungen dürften zugleich beweisen, wie willkürlich die von Abendländern verwendeten Termini „Pantheismus“ und „Polytheismus“ eigentlich sind.

Obgleich man dann und wann eine andere Auffassung von einem Worte oder einer Wendung im Texte hat als die die Radhakrishnan vertritt, finde ich seine Erklärung des Gottesbegriffes zutreffend und zwar nicht nur hinsichtlich der Upanișaden sondern des Brahmanismus-Hinduismus im allgemeinen: „The Supreme is not so much an immanent God as an experienced God"'1.

${ }^{1}$ The Principal Upanișads, S. 142. 